\title{
Immunsuppression und Infektionsprophylaxe nach Lungentransplantation
}

\author{
Immunosuppression and Infection Prophylaxis after Lung Transplantation
}

Autoren

Institut

\section{Neurohr, J. Behr}

Schwerpunkt Pneumologie (Leiter Prof. Dr. J. Behr), Medizinische Klinik und Poliklinik I Campus Großhadern (Direktor Prof. Dr. G. Steinbeck), Klinikum der Universität München eingereicht 13.7.2010

akzeptiert 16.7.2010

Bibliografie

DoI http://dx.doi.org/

10.1055/s-0030-1255762

Online-Publikation: 19. 10. 2010

Pneumologie 2011; 65:

94-102 (c) Georg Thieme

Verlag KG Stuttgart · New York

ISSN 0934-8387

Korrespondenzadresse

Dr. med. Claus Neurohr

Medizinische Klinik und

Poliklinik I

Klinikum der Universität

München - Großhadern

Marchioninistr. 15

81377 München

claus.neurohr@med.uni-

muenchen.de

Serienherausgeber

C. Kroegel, Jena

T. Welte, Hannover

\section{Zusammenfassung}

$\nabla$

Die Lungentransplantation (LTX) ist ein etabliertes Therapieverfahren für Lungenerkrankungen im Endstadium. Hauptursachen für die unbefriedigenden Langzeitüberlebensraten sind Infektionen und das Bronchiolitis-obliterans-Syndrom (BOS). Für beide Komplikationen spielt eine optimale immunsuppressive Strategie eine entscheidende Rolle. Über 60\% der Organempfänger erhalten eine Induktionstherapie, ohne dass bisher ein signifikanter Vorteil bezüglich Langzeitüberleben in kontrollierten Studien demonstriert wurde. Die große Mehrheit der Patienten wird lebenslang mit einer dreifach immunsuppressiven Erhaltungstherapie, bestehend aus einem Calcineurin-Inhibitor, einem Zellzyklus-Inhibitor und einem oralen Kortikosteroid, behandelt. Eine eindeutige Überlegenheit in Bezug auf Langzeitüberleben konnte bisher jedoch für keine der spezifischen Immunsuppressiva-Kombination gezeigt werden. Ob die neueren Proliferations-Signal-Inhibitoren Sirolimus und Everolimus signifikante Vorteile bieten, ist noch nicht abschließend zu beurteilen. Zur Therapie des BOS werden eine Umstellung der Erhaltungstherapie, eine erneute Induktionstherapie, inhalatives Cyclosporin A sowie u.a. Azithromyzin, extrakorporale Photopherese und Lymphsystembestrahlung angewendet. Die Infektionsprophylaxe nach LTX spielt eine entscheidende Rolle bei der Vermeidung von akuten Komplikationen und auch in der Verhinderung des BOS. Insbesondere zur Prophylaxe einer Pneumocystis- und Cytomegalievirus-Erkrankung stehen effektive medikamentöse Optionen zur Verfügung. Darüber hinaus wurde auch die Kolonisierung mit Pseudomonas aeruginosa und Aspergillus-Spezies als Risikofaktor für BOS erkannt. Entsprechende prophylaktische bzw. präemptive Therapieansätze finden daher in unterschiedlichem Ausmaß in den meisten Transplantationszentren Anwendung.

\section{Abstract \\ $\nabla$}

Lung transplantation (LTX) is an established therapeutic option for end-stage lung diseases. The main reasons for limited long-term survival rates are infections and bronchiolitis obliterans syndrome (BOS). An optimal immunosuppressive regimen is of critical importance for the prevention of both complications. Induction therapy is used in approximately $60 \%$ of recipients. However, there are no controlled trials demonstrating a significant long-term survival benefit. The vast majority of patients receive a triple maintenance immunosuppressive therapy consisting of a calcineurin-inhibitor, a cell cycle inhibitor and corticosteroids. So far, no specific immunosuppressive drug combination has proven superiority regarding long-term survival rates. The potential benefits of the proliferation signal inhibitors sirolimus and everolimus remain to be elucidated. Therapeutic options for BOS encompass a switch in maintenance therapy, renewed induction therapy, aerolised cyclosporine, azithromycine, extracorporeal photopheresis and total lymphoid irradiation. Infection prophylaxis after LTX plays a pivotal role to guard against acute complications and for the prevention of BOS. In particular, prophylaxis for pneumocystis and cytomegalovirus disease is very effective. Moreover, colonisation with Pseudomonas aeruginosa and Aspergillus spp. was identified as risk factor for BOS. Consequently, in most transplant centres prophylactic and pre-emptive therapeutic approaches are applied in varying degrees. 


\section{Einleitung}

\section{$\nabla$}

Die Lungentransplantation (LTX) hat sich zu einem etablierten Therapieverfahren für Patienten mit Lungenerkrankungen im Endstadium entwickelt. Leider können die transplantierten Patienten mit einer mittleren Überlebensrate von 52\% nach 5 Jahren nicht als geheilt gelten. Die beiden Haupttodesursachen im Langzeitverlauf nach LTX sind die Bronchiolitis obliterans (BO) mit der klinischen Manifestation als Bronchiolitis-obliteransSyndrom (BOS) sowie pulmonale Infektionen [1]. Die BO wird zwar als chronische Form der Abstoßung verstanden, ist aber in ihrer Ätiologie nicht geklärt. Sowohl stattgehabte akute Abstoßungen als auch Infektionen wurden jedoch als Hauptrisikofaktoren für das spätere Auftreten eines BOS identifiziert. Die Optimierung der immunsuppressiven Strategie und die Verhinderung von Infektionen sind daher sicher die wichtigsten Beiträge zur Verbesserung der Langzeitergebnisse. Diese Übersicht schildert den aktuellen Wissensstand zu immunsuppressiven Therapiestrategien nach LTX und erörtert die Optionen einer rationalen Infektionsprophylaxe.

\section{Induktionstherapie}

$\nabla$

\section{Rationale der Induktionstherapie}

Ziel der Induktionstherapie ist eine möglichst rasche und weitgehende Immunsuppression unmittelbar nach Transplantation. Dadurch sollen Interaktionen zwischen dem Immunsystem des Empfängers und den Antigenen des Transplantats möglichst gering gehalten werden. Ziel ist es, sowohl die Rate akuter Abstoßungsreaktionen als auch eine vermutete, durch die frühe Alloreaktivität ausgelöste chronische Entzündungsreaktion zu reduzieren.

Die Daten der International Society for Heart and Lung Transplantation (ISHLT) zeigen, dass, bei steigender Tendenz, im Jahre 2008 ca. 62\% der LTX-Patienten eine Induktionstherapie erhielten. Angriffspunkt sind die aktivierten Lymphozyten des Transplantatempfängers. Hierbei ist in den letzen Jahren eine Entwicklung weg von den polyklonalen Anti-Lymphozyten (ALG)- bzw. Anti-Thymozyten (ATG)-Präparationen (ca. 10\%) und Anti-CD3 (OKT3)-basierten Schemata (ca. 3\%) hin zur Verwendung von monoklonalen Ansätzen mit Interleukin (IL)-2-Rezeptor-Antagonisten (ca. 44\%) und Anti-CD52-Antikörpern (ca. 18\%) zu verzeichnen [1]. Sowohl die Dosierung als auch die Dauer der Therapie wird in den verschiedenen Zentren sehr unterschiedlich gehandhabt. Somit ist der Vergleich verschiedener Studien als auch eine Interpretation des ISHLT-Registers deutlich erschwert. Daher ist der exakte Stellenwert der Induktionstherapie bezüglich der Rate akuter Abstoßungen, aber auch der Inzidenz von Infektionen und maligner Tumoren weiterhin unklar. Aktuelle ISHLT-Daten deuten an, dass eine Induktionstherapie einen kleinen, aber signifikant positiven Effekt auf das Langzeitüberleben nach LTX hat [1] ( $\bullet$ Tab. 1).

\section{Polyklonale Anti-Lymphozyten (ALG) und}

Anti-Thymozyten (ATG) Antikörper

ALG/ATG werden aus Pferde $\left(\right.$ Atgam $\left.^{\circledR}\right)$ - oder Kaninchenserum (Thymoglobulin ${ }^{\circledR}$ ) nach Injektion von humanen Thymozyten oder Lymphozyten gewonnen. Die Antikörper sind gegen zahlreiche Oberflächenantigene gerichtet, die z.T. wie CD3, CD4 und CD8 nur auf T-Zellen präsentiert werden, wohingegen andere wie die Adhäsionsmoleküle CD11b und CD18 auch auf anderen
Zellen nachweisbar sind. Es kommt zu einer schnellen und ausgeprägten Lymphopenie über Fc-Rezeptor-abhängige Mechanismen wie Komplement- oder Zell-vermittelte Zytolyse sowie Phagozytose durch Makrophagen. Typische Nebenwirkungen sind allergische Reaktionen und Allgemeinsymptome durch die Zytokinfreisetzung.

In einer älteren Studie führte die Therapie mit ALG/ATG im Vergleich zu einem Regime ohne Induktionstherapie zu einer signifikanten Reduktion der Rate an akuten Abstoßungsperioden. Eine Verbesserung der medianen Überlebensraten oder eine signifikante Verminderung der Inzidenz des BOS war jedoch auch unter Berücksichtigung der aktuellen ISHLT-Daten nicht zu verzeichnen [1-3].

Monoklonaler Anti-CD3-Antikörper Muromonab (OKT3) Muromonab-CD3 (Orthoclone OKT3reg) ist ein monoklonaler Maus-Antikörper gegen den CD3-Komplex, der mit dem T-Lymphozyten-Antigen-Rezeptor assoziiert ist. OKT3 induziert eine Depletion zirkulierender T-Lymphozyten. Obwohl es Hinweise gibt, dass OKT3 die Inzidenz akuter und chronischer Abstoßungsreaktionen reduziert, findet es nur in sehr begrenztem Umfang Anwendung. Gründe hierfür sind die z.T. ausgeprägte ZytokinFreisetzung, die zur hämodynamischen Instabilität führen kann, sowie eine erhöhte Frequenz bakterieller Infektionen [4-6].

\section{Monoklonale Anti-Interleukin-2-Rezeptor-Antikörper Daclizumab und Basiliximab}

Daclizumab (Zenapax ${ }^{\circledR}$ ) und Basiliximab (Simulec ${ }^{\circledR}$ ) sind chimäre, humanisierte monoklonale Antikörper, die spezifisch die $\alpha$-Untereinheit des Interleukin (IL)-2-Rezeptors (CD25) blockieren. Im Gegensatz zu ALG/ATG inhibieren sie dadurch die T-ZellProliferation und -Differenzierung, ohne dass es zu T-Zell-Depletion kommt. Konzeptionell sind daher die monoklonalen Antikörper bezüglich der Steuerbarkeit, Verträglichkeit, Spezifität und Reproduzierbarkeit dem polyklonalen Ansatz überlegen. Im Vergleich zu Kontrollgruppen ohne Induktionstherapie zeigte sich unter Therapie mit Daclizumab eine signifikant niedrigere Rate von akuten Abstoßungsepisoden ohne erhöhte Inzidenz von Infektionen. Trotz Hinweisen, dass Daclizumab bei BOS eine Verlangsamung des Progresses bewirken kann, ergab sich in größer angelegten Studien und aus den Daten des ISHLT-Registers kein Hinweis auf einen Überlebensvorteil oder eine geringere BOS-Inzidenz eines spezifischen Induktionsschemas [7-9].

\section{Monoklonaler Anti-CD52-Antikörper Alemtuzumab}

Alemtuzumab (Campath- $1 \mathrm{H}^{\circledR}$ ) ist gegen das CD52-Antigen gerichtet, welches auf der Oberfläche von B- und T-Lymphozyten, natürlichen Killerzellen (NK) sowie anderen immunkompetenten Zellen exprimiert wird. Der genaue Wirkmechanismus ist nicht vollständig verstanden, aber die Gabe von Alemtuzumab führt zu einer lang anhaltenden (> 12 Monate) Lymphozytendepletion. Für die Lungentransplantation scheint Alemtuzumab eine alternative Induktionstherapie darzustellen, die eine Kombination mit einer dosisreduzierten Erhaltungstherapie ohne erhöhte Rate akuter Abstoßungen oder Infektionen bzw. lymphoproliferativer Erkrankungen (PTLD) erlauben könnte. Hierzu fehlen jedoch kontrollierte Langzeitstudien bezüglich Überleben und BOS $[10,11]$. 


\section{Erhaltungstherapie}

$\nabla$

Die Einführung von Cyclosporin A (CyA) war ein Meilenstein zum klinischen Erfolg der LTX. Ohne Cyclosporin A endeten die LTX unvermeidlicherweise in einem Misserfolg, da die bis dahin verwendete Immunsuppression aus Azathioprin und hochdosierten Kortikosteroiden entweder zu ausgeprägten Wundheilungsstörungen im Bereich der Anastomosen führte oder nicht ausreichte, um akute Abstoßungen zu kontrollieren.

Die immunsuppressive Erhaltungstherapie nach LTX zielt insbesondere auf die Verhinderung akuter zellulärer Transplantatabstoßungsreaktionen im Langzeitverlauf ab. Im Gegensatz zur Nachsorge nach Transplantation anderer solider Organe erhält die Mehrheit der Patienten nach LTX eine lebenslange dreifach immunsuppressive Therapie (sog. Triple-Therapie) [1]. Sie besteht in der Regel aus einem Calcineurin-Inhibitor (CyA oder Tacrolimus), einem Zellzyklus-Inhibitor bzw. Anti-Metabolit (Azathioprin oder Mycophenolat Mofetil) und Kortikosteroiden. Die Kombination von unterschiedlichen Medikamenten mit simultaner Beeinflussung von verschiedenen Signaltransduktionswegen zielt auf eine ausreichende Immunsuppression bei gleichzeitiger Minimierung der unerwünschten Nebenwirkungen ab $[12,13]$. Mit den Proliferations-Signal-Inhibitoren Sirolimus und Everolimus steht eine vierte Gruppe neuerer Immunsuppressiva zur Verfügung und erlaubt eine Reihe weiterer unterschiedlicher Kombinationen ( $\bullet$ Tab. 1$)$.

\section{Calcineurin-Inhibitoren: Ciclosporin, Tacrolimus (FK506) Cyclosporin A (Ciclosporin)}

Mit der Entdeckung der immunsuppressiven Eigenschaften von Cyclosporin A (CyA) 1976 und seiner Markteinführung 1983 begann eine neue Epoche der Transplantationsmedizin [14]. CyA ist ein aus dem Schlauchpilz Tolypocladium inflatum isoliertes zyklisches Peptid. Es bildet intrazelluläre Komplexe mit zytoplasmatischen Proteinen aus der Gruppe der Cyclophiline, welche ihrerseits an Calcineurin binden. Calcineurin ist für die Dephosphorylierung von Gen-regulierenden Proteinen wie NF-AT (nuclear factor of activated T-Cells) verantwortlich. Die CyA-Cyclophilin-Komplexe blockieren die enzymatische Aktivität von Calcineurin und inhibieren somit die Transkription von verschiedenen Zytokinen wie Interleukin (IL)-2, -3, -4, -5, Interferon (IFN)- $\gamma$, tumor necrosis factor (TNF)- $\alpha$ und granulocyte/macrophage colony stimulating factor (GMCSF). Die in Zellen des Immunsystems im Vergleich zu nicht-immunkompetenten Zellen verminderte Aktivität von Calcineurin und die Tatsache, dass Calcineurin für die Aktivierung von T-Zellen von entscheidender Bedeutung ist, erklären die T-Zell-spezifische Wirkung von CyA.

CyA ist eine stark lipophile Substanz mit ausgeprägter Variabilität der enteralen Absorption bei niedriger oraler Bioverfügbarkeit. Durch die Einführung einer Formulierung von CyA in einer Mikroemulsion 1996 wurden eine raschere und höhere orale Bioverfügbarkeit sowie eine geringere intraindividuelle Variabilität der Blutkonzentration erreicht.

Die Dosisfestlegung von CyA erfolgt in der Praxis individuell nach Medikamentenspiegelbestimmung (therapeutic drug monitoring) nach Messung morgendlicher Talspiegel $\left(C_{0}\right)$. Verschiedene Studien haben jedoch gezeigt, dass Talspiegel $\left(C_{0}\right)$ nur eine unbefriedigende Korrelation mit der tatsächlichen Medikamentenexposition (AUC - area under the curve/total drug exposure), die mithilfe eines pharmakokinetischen Profils über 12 Stunden (AUC $_{1-12}$ ) bestimmt wird, aufweisen. Bei nierentransplantierten Patienten geht dabei eine niedrige systemische CyA-Exposition, bestimmt mittels $\mathrm{AUC}_{1-12}$, mit einem erhöhten Risiko für akute Abstoßungsreaktionen und chronischem Transplantatversagen einher, wobei bei starker CyA-Exposition eine deutliche Nephrotoxizität besteht. Da die Bestimmung der tatsächlichen Medikamentenexposition $\left(\mathrm{AUC}_{1-12}\right.$ ) wegen des großen logistischen Aufwandes für die Routinenachsorge nicht geeignet ist, wurde mit der Bestimmung des CyA-Spiegels zwei Stunden nach der morgendlichen Medikamenteneinnahme $\left(C_{2}\right)$ ein Parameter gefunden, der gut mit der $\mathrm{AUC}_{1-12}$ korreliert. Für die Nierentransplantation konnte gezeigt werden, dass CyA-Dosisfestlegung mittels $\mathrm{C}_{2}$-Monitoring der Verwendung von Talspiegeln $\left(\mathrm{C}_{0}\right)$ zur Vermeidung akuter Abstoßungsreaktionen und chronischen Transplantatverlusts überlegen ist. Vor diesem Hintergrund wird das $\mathrm{C}_{2^{-}}$ Monitoring nach Nieren- und Lebertransplantation empfohlen und entsprechende Zielspiegel wurden etabliert [14-16].

Auch für die Lungentransplantation gibt es Hinweise, dass die Verwendung von $\mathrm{C}_{2}$-Spiegeln mit einer Reduzierung der Rate akuter Abstoßungen und BOS einhergehen. Die in diesen Studien verwendeten $C_{2}$-Zielspiegel orientierten sich dabei jeweils an den vorliegenden Daten der anderen soliden Organtransplantationen, da formale Empfehlungen für Patienten nach LTX zum jetzigen Zeitpunkt nicht vorliegen $[17,18]$. Häufigste Nebenwirkung von CyA ist die dosisabhängige und nur im Frühstadium (teil)reversible Nephrotoxizität bis hin zur Dialysepflichtigkeit, wie sie für Calcineurin-Inhibitoren typisch ist. Darüber hinaus sind arterielle Hypertonie, Dyslipidämie, Hirsutismus, Gingivahyperplasie und neurologische Komplikationen bis hin zu Krampfanfällen mögliche Folgen einer Dauertherapie [19].

\section{Tacrolimus (FK506)}

Tacrolimus (Prograf ${ }^{\circledR}$ ) wurde 1984 entdeckt und ist ein Makrolid, welches aus dem Bakterium Streptomyces tsukubaensis isoliert wird. Es entfaltet seine immunsuppressive Wirkung über einen ähnlichen Mechanismus wie Cyclosporin A. Tacrolimus (FK506), assoziiert im Zytoplasma an sogenannte FK-bindende Proteine (FKBPs), die ihrerseits dann die enzymatische Aktivität von Calcineurin inhibieren. In vitro ist Tacrolimus aufgrund höherer Bindungsaffinität von Tacrolimus an FKBPs ca. 50 - 100-mal potenter als CyA [16].

Tacrolimus hat, wie CyA, eine enge therapeutische Breite und insbesondere nephrotoxische Nebenwirkungen bei allerdings geringerer Neigung, eine arterielle Hypertonie oder eine Hyperlipidämie zu induzieren. Die Metabolisierung von Tacrolimus durch Cytochrom-P450-Enzyme beginnt bereits in der Darmwand und bedingt eine Vielzahl von Interaktionen mit anderen Medikamenten mit der Notwendigkeit zur zeitnahen Dosisanpassung.

Ähnlich wie bei CyA erfolgt die Dosisfestlegung von Tacrolimus individuell nach Medikamentenspiegelbestimmung. In der klinischen Routine werden in der Regel morgendliche Talspiegel $\left(C_{0}\right)$ bestimmt. Auch für Tacrolimus gibt es Hinweise, dass $\mathrm{C}_{0}$-Spiegel nur eine sehr ungenaue Aussage über die tatsächliche Medikamentenexposition $\left(\mathrm{AUC}_{1-12}\right)$ erlauben und kein optimales Mittel zur Steuerung der Dosis darstellen. Entsprechende Studien legen nahe, dass mittels Spiegelbestimmung nach Medikamenteneinnahme (sog. postdose-monitoring) eine effektivere und nebenwirkungsärmere Anpassung der Tacrolimusdosis gewährleistet werden könnte $[20,21]$. Mit Advagraf ${ }^{\circledR}$ steht darüber hinaus eine Tacrolimus-Formulierung zur Verfügung, die nur eine einmalige tägliche Gabe notwendig macht. Allerdings existieren zum jetzigen Zeitpunkt keine publizierten Daten zur Verwendung dieses Präparates nach LTX. 
Vergleich von Cyclosporin A und Tacrolimus

In den letzten 10 Jahren hat es einen deutlichen Trend zur Verwendung von Tacrolimus statt CyA als Standard-CalcineurinInhibitor gegeben. Laut ISHLT ist Tacrolimus sowohl im ersten als auch im fünften Jahr nach LTX der am häufigsten eingesetzte Calcineurin-Inhibitor [1].

Eine Reihe von Studien haben die Wirksamkeit von CyA und Tacrolimus nach LTX verglichen. Dabei hat sich Tacrolimus als effektives Medikament in Kombination sowohl mit Azathioprin als auch Rapamycin und Mycophenolat Mofetil etabliert. Tacrolimus-basierte Therapieregime gehen dabei, bei mit CyA vergleichbarer Nephrotoxiziät, nicht mit einem erhöhten Risiko für maligne Tumoren oder Infektionen einher [14,22]. Tacrolimus ist mit weniger Störungen des Lipidstoffwechsels und einer geringeren Rate an arterieller Hypertension assoziiert. Darüber hinaus spielen Hirsutismus und Gingivahyperplasie als Nebenwirkungen keine relevante Rolle, während die prodiabetogene Potenz von Tacrolimus höher als die von CyA zu sein scheint.

Zum jetzigen Zeitpunkt gibt es keine kontrollierten Studien, die einen Vorteil bezüglich des Überlebens für Tacrolimus im Vergleich zu CyA demonstrieren. Mehrere Untersuchungen weisen jedoch daraufhin, dass Tacrolimus die Rate akuter Abstoßungsreaktionen reduziert und auch zu einer Verminderung der Inzidenz von BOS führt. Insbesondere die Kombination mit Mycophenolat Mofetil scheint diesbezüglich die größten Vorteile zu bieten [1,23-27].

\section{Zellzyklus-Inhibitoren: Azathioprin, Mycophenolat Mofetil \\ Azathioprin}

Azathioprin inhibiert die de novo Purinsynthese und hemmt so die Bildung sowohl von DNA als auch RNA mit einem antiproliferativen Effekt auf T- und B-Lymphozyten, jedoch ohne die Zytokinproduktion zu beeinflussen. Die Entwicklung von Azathioprin stellte den ersten Durchbruch in der Transplantationsmedizin dar. In Kombination mit Cyclosporin A bildet Azathioprin die Grundlage der klassischen immunsuppressiven Triple-Therapie nach LTX. Hiermit konnten im Vergleich zur Therapie mit CyA und Steroid alleine niedrigere Raten akuter und chronischer Abstoßungen erreicht werden $[13,28]$.

\section{Mycophenolat Mofetil (MMF)}

Mycophenolat Mofetil (MMF, CellCept ${ }^{\circledR}$ ) ist das Propharmakon der Mycophenolsäure (MPA). MPA ist ein selektiver, nicht-kompetitiver und reversibler Hemmer der Inosinmonophosphat-Dehydrogenase (IMPDH), dem limitierenden Enzym der Purin-denovo-Synthese. Da für die Proliferation von T- und B-Lymphozyten die De-novo-Synthese von Purinen unerlässlich ist, während andere Zellarten den Wiederverwertungsstoffwechsel (salvagepathway) benützen können, wirkt MPA selektiv stärker zytostatisch auf sich teilende B- und T-Lymphozyten. Darüber hinaus zeigen experimentelle Daten anti-proliferative Eigenschaften für MMF, die einen potenziell positiven Effekt für eine fibrosierende Erkrankung wie das BOS erwarten lassen [29].

Nach LTX findet MMF in den letzten Jahren zunehmend stärkere Verwendung. 1999 erhielten ein Jahr nach LTX lediglich 20\% der Patienten MMF, während der Anteil 2005 bereits bei 46\% lag $[1,14]$. In randomisierten, plazebokontrollierten Studien konnte gezeigt werden, das MMF im Vergleich mit Azathioprin zu einer signifikanten Reduktion von akuten Abstoßungen nach Nierenund Herztransplantation führt [30,31]. Während kleinere, nichtkontrollierte Studien einen ähnlichen positiven Effekt auch nach
LTX zu belegen schienen, konnten zwei prospektive, randomisierte Untersuchungen keine signifikanten Vorteile für MMF gegen Azathioprin jeweils in Kombination mit Cyclosporin A bezüglich akuten Abstoßungsreaktionen, Infektionsraten, Überleben und BOS demonstrieren. Wie bereits erwähnt, scheinen die Daten des ISHLT-Registers nahezulegen, dass die Kombination MMF mit Tacrolimus zumindest die Anzahl behandelter AbstoBungen im 1. Jahr nach LTX reduziert, ohne dass hierzu jedoch kontrollierte Studien vorliegen $[1,32,33]$. Ähnlich wie für CyA und Tacrolimus gilt auch für MMF, dass aufgrund hoher interund intraindividueller Variabilität der Pharmakokinetik sowohl die Gabe von MMF in einer festen Dosierung als auch das Monitoring mittels Talspiegelbestimmung noch nicht die optimalen Methoden zur Dosisanpassung darstellen.

Knochenmarkstoxizität und gastrointestinale Beschwerden stellen die häufigsten Nebenwirkungen von MMF dar und bedingen in der Praxis in vielen Fällen eine Reduktion oder sogar ein zeitweises Beendigen der Therapie. Insbesondere früh nach Transplantation kommt es bei gleichzeitiger Cytomegalievirus-Reaktivierung und entsprechender antiviraler Behandlung fast regelhaft zu einer Leukopenie, sodass eine Verminderung der MMFDosis zu einer unerwünschten Verminderung der immunsuppressiven Therapie zwingt.

Mit Myfortic ${ }^{\circledR}$ steht eine magensaftresistente Formulierung der Mycophenolsäure zur Verfügung, die durch Reduktion der oberen gastrointestinalen Nebenwirkungen eine verbesserte Verträglichkeit bei gleicher Wirksamkeit erreichen kann.

\section{Proliferations-Signal-Inhibitoren Sirolimus und Everolimus \\ Sirolimus (Rapamycin)}

Sirolimus und Everolimus gehören zur Gruppe der sog. mTOR (mammalian Target of Rapamycin)-Inhibitoren. Sirolimus (Rapamune ${ }^{\circledR}$ ) ist ein aus dem Pilz Streptomyces hygroscopius isoliertes Makrolid. Der immunsuppressive und antiproliferative Effekt von Sirolimus basiert auf der Inhibierung der IL-2-induzierten TZell-Proliferation mit Blockade des Zellzyklus beim Übergang von der G1- in die S-Phase. Erfahrungen mit anderen soliden Organtransplantationen zeigen, dass Sirolimus eine effektive Form der Immunsuppression darstellt. Insbesondere das Fehlen einer relevanten Nephrotoxizität lässt es zu einer Alternative zu Calcineurin-Inhibitoren bei progredienter Niereninsuffizienz werden $[34,35]$.

Auch nach LTX wird Sirolimus bereits bei einer Minderheit der Patienten eingesetzt (8\% nach 1 Jahr nach LTX, 16\% im 5. Jahr nach LTX [1]. Kleine, unkontrollierte Studien weisen darauf hin, dass sich auch in diesem Patientengut nach Umstellung von einem Calcineurin-Inhibitor auf Sirolimus eine vorbestehende Niereninsuffizienz wieder verbessern kann [36-38]. Zusätzlich stellt Sirolimus auch eine mögliche Option bei rezidivierenden akuten Abstoßungen oder progredientem BOS dar, ohne dass hierzu kontrollierte Studien vorliegen würden $[39,40]$. Trotz dieser positiven Effekte ist ein verstärkter Einsatz von Sirolimus nach LTX aus verschiedenen Gründen kritisch zu bewerten. Zum einen scheint der Einsatz von Sirolimus als primäre Immunsuppression mit einer erhöhten Rate an schweren Wundheilungsstörungen der bronchialen Anastomose einherzugehen [41,42]. Zum anderen gibt es Hinweise, dass lebensbedrohliche Sirolimus-induzierte, interstitielle Pneumonitiden oder pulmonale Vaskulitiden als Ausdruck einer pulmonalen Toxizität eine klinisch relevante Komplikation darstellen [43]. Die bisher nur in Abstractform publizierten Ergebnisse des randomisierten, multi- 


\begin{tabular}{|c|c|c|}
\hline Medikament & Wirkmechanismus & Nebenwirkungen \\
\hline ALG/ATG & $\begin{array}{l}\text { Blockade von Antigenen auf lymphoiden Zellen, } \\
\text { Depletion zirkulierender Lymphozyten }\end{array}$ & $\begin{array}{l}\text { Zytokin-Freisetzungssyndrom, } \\
\text { Leukopenie }\end{array}$ \\
\hline OKT3 & $\begin{array}{l}\text { Monoklonaler Anti-CD3-Antikörper, } \\
\text { Depletion zirkulierender Lymphozyten }\end{array}$ & $\begin{array}{l}\text { Zytokin-Freisetzungssyndrom, } \\
\text { bakterielle Infektionen } \uparrow\end{array}$ \\
\hline $\begin{array}{l}\text { Daclizumab/ } \\
\text { Basiliximab }\end{array}$ & $\begin{array}{l}\text { Bindung an die } \alpha \text {-Kette des IL-2-Rezeptors, } \\
\text { Blockade der IL-2-induzierten T-Zell-Aktivität }\end{array}$ & (Pilz) Infektionen $\uparrow$ \\
\hline Alemtuzumab & $\begin{array}{l}\text { Monoklonaler Anti-CD52-Antikörper, } \\
\text { Depletion zirkulierender Lymphozyten }\end{array}$ & Infektionen $\uparrow$ \\
\hline Cyclosporin A & $\begin{array}{l}\text { Bindung an Cyclophylin, Inhibierung von } \\
\text { Calcineurin, Blockierung der Gen-Transkription } \\
\text { von Zytokinen }\end{array}$ & $\begin{array}{l}\text { Nephrotoxizität, arterielle Hyper- } \\
\text { tonie, Hypercholesterinämie, u. a. }\end{array}$ \\
\hline Tacrolimus & $\begin{array}{l}\text { Bindung an FKBP, Inhibierung von Calcineurin, } \\
\text { Blockierung der Gen-Transkription von Zytokinen }\end{array}$ & $\begin{array}{l}\text { Nephrotoxizität, Neurotoxizität, } \\
\text { Diabetes mellitus, u. a. }\end{array}$ \\
\hline Azathioprin & $\begin{array}{l}\text { Inhibierung der Purinbiosynthese und } \\
\text { Lymphozytenproliferation }\end{array}$ & Leukopenie \\
\hline Mycophenolat Mofetil & $\begin{array}{l}\text { Inhibierung der Purinbiosynthese und } \\
\text { Lymphozytenproliferation }\end{array}$ & Diarrhoe \\
\hline Sirolimus/Everolimus & Blockade der IL-2-induzierten T-Zell-Proliferation & $\begin{array}{l}\text { Wundheilungsstörungen der Anas- } \\
\text { tomosen, interstitielle Pneumonitis }\end{array}$ \\
\hline Azithromycin & nicht geklärt & Übelkeit, Diarrhoe (selten) \\
\hline
\end{tabular}

Tab. 1 Immunsuppressiva Wirkmechanismen und Nebenwirkungen. zentrischen AIRSAC-Trials ergaben bei einer mittleren Nachverfolgungszeit von 2,7 Jahren keine Unterschiede bezüglich BOS und Überleben für Sirolimus im Vergleich zu Azathioprin jeweils in Kombination mit Tacrolimus und Prednisolon bei jedoch erhöhter Rate von vorzeitigen Therapieabbrüchen und Infektionen im Sirolimus-Arm [44].

\section{Everolimus}

Everolimus (Certican ${ }^{\circledR}$ ) ist ein synthetisch hergestelltes Sirolimus-Derivat mit verbesserter Bioverfügbarkeit und sowohl immunsuppressiven als auch anti-proliferativen Eigenschaften. Insbesondere nach Herz-Transplantation konnte durch Everolimus die Inzidenz und der Schweregrad der Transplantatvaskulopathie reduziert werden. Im Tierexperiment zeigte sich auch ein positiver Effekt auf die Bronchiolitis obliterans nach LTX [45]. In einer randomisierten, doppelblinden Vergleichstudie mit 213 Patienten länger als 3 Monate nach LTX mit Azathioprin versus Everolimus, jeweils in Kombination mit Cyclosporin A, war Everolimus bezüglich der Rate akuter Abstoßungen und der Inzidenz von BOS nach 12 bzw. 24 Monaten überlegen. Medikamentinduzierte Pneumonitiden wurden hierbei nicht beobachtet, wobei jedoch signifikant häufiger bakterielle Infektionen und Studienabbrüche aufgrund von Nebenwirkungen im Everolimus-Arm zu verzeichnen waren [46]. Die 1-Jahres-Interimsanalyse einer prospektiven, randomisierten Studie ergab für Everolimus im Vergleich mit MMF jeweils in Kombination mit CyA und Steroiden sogar einen signifikanten Überlebensvorteil [47]. Darüber hinaus konnte in einer kürzlich publizierten multizentrischen, randomisierten Studie gezeigt werden, dass bei Gabe von Everolimus eine relevante Verminderung der Calcineurin-Inhibitordosis und damit eine signifikante Verbesserung bei vorbestehend eingeschränkter Nierenfunktion erreicht werden kann [48]. Gleichwohl gibt es auch für Everolimus Fallberichte über eine relevante pulmonale Toxizität und thrombotische Mikroangiopathien bis hin zum hämolytisch-urämischen Syndrom (HUS) [49,50].

\section{Kortikosteroide}

Typischerweise werden nach LTX in der frühen postoperativen Phase intravenöse Kortikosteroide entsprechend einer Dosis von ca. 0,5-1,5 mg Methylprednisolon/kg Körpergewicht verwendet und dann auf eine orale Erhaltungsdosis schrittweise reduziert. Die Daten des ISHLT-Registers zeigen, dass im Gegensatz zu anderen soliden Organtransplantationen ca. 95\% der Patienten nach LTX auch nach einem und nach fünf Jahren noch Kortikosteroide erhalten [1]. Obwohl dieses Therapieregime mit signifikanten Nebenwirkungen assoziiert ist und es auch für Kortikosteroide Hinweise gibt, dass eine Medikamentendosierung mittels Spiegelbestimmung sinnvoll scheint, fehlen kontrollierte Studien zur systematischen Steroidreduktion und steroidfreien Immunsuppression [51]. Beobachtungen an Patienten mit langfristig stabilem Verlauf zeigen, dass eine steroidfreie Immunsuppression auch nach LTX möglich ist, ohne den Transplantationserfolg zu gefährden. Darüber hinaus stellen sich positive Effekte für Begleiterkrankungen wie Diabetes mellitus, arterielle Hypertonie und Hypercholesterinämie auch bei Fortführung der übrigen Immunsuppressiva ein [52,53].

\section{Weitere immunmodulierende Therapieoptionen}

\section{Lymphsystembestrahlung (Total lymphoid irradiation)} Bestrahlung von lymphatischem Gewebe führt theoretisch $\mathrm{zu}$ einer verstärkten Immunsuppression zusätzlich zur medikamentenbasierten Unterdrückung der körpereigenen Abwehr. Nach Herztransplantation konnte gezeigt werden, dass eine Lymphsystembestrahlung (Total lymphoid irradiation, TLI) einen positiven Effekt bei rezidivierenden akuten Abstoßungsreaktionen zu haben scheint [54]. Nach LTX untersuchten bisher drei unkontrollierte Studien eine Lymphsystembestrahlung bei progredientem BOS [55-57). Die Progredienz der Lungenfunktionsverschlechterung wurde hierbei jeweils reduziert, wobei dieses recht aufwendige Therapieverfahren auch mit einer deutlichen Nebenwirkungsrate bei den meist schon fortgeschritten erkrankten Patienten einhergeht. 


\section{Extrakorporale Photopherese (ExP)}

Die extrakorporale Photopherese (ExP) beinhaltet eine Leukopherese und Inkubation mit dem photosensitiven Molekül 8-Methoxypsoralen in Kombination mit Bestrahlung durch Ultraviolett-(UVA)-Licht. Der Mechanismus der Immunmodulation ist nur ansatzweise verstanden und scheint z.T. darauf zu beruhen, dass lichtaktiviertes Psoralen zu einem DNA-Crosslinking der behandelten Leukozyten mit konsekutiver Zellzerstörung führt. Nach LTX wurde die extrakorporale Photopherese in verschiedenen kleinen, retrospektiven bzw. unkontrollierten Studien bei BOS eingesetzt und hat in einigen Fällen zu einer Stabilisierung des FEV1 oder zu einer Verlangsamung des FEV1-Verlustes geführt [58-60]. Zum jetzigen Zeitpunkt fehlen prospektive, randomisierte Studien, die eine genaue Bewertung dieses in der Regel sehr nebenwirkungsarmen Therapiekonzeptes erlauben.

\section{Azithromyzin}

Azithromyzin ist ein Makrolidantibiotikum, das neben einer direkten antibakteriellen Wirkung auch erregerunabhängige antiinflammatorische Effekte sowohl in vitro als auch in vivo besitzt. Da neben fibrotischen vor allem auch entzündliche Veränderungen der kleinen Atemwege ein typisches Merkmal der Bronchiolitis obliterans sind, ergibt sich hier eine Rationale zum Einsatz auch bei BOS nach LTX. In mehreren kleineren unkontrollierten Studien konnte gezeigt werden, dass es bei BOS unter Azithromyzin bei einem Teil der Patienten zu einer Stabilisierung und in einigen Fällen auch zu einer Verbesserung der Lungenfunktion kam [61,62]. Insbesondere der Nachweis einer Neutrophilie in der bronchoalveolären Lavageflüssigkeit scheint hierbei Patienten zu identifizieren, bei denen eine Reversibilität des Lungenfunktionsverlustes möglich ist [63,64]. Zur Klärung der Frage, ob eine prophylaktische Azithromyzin-Gabe nach LTX zur Therapie bzw. Prävention des BOS im Frühstadium sinnvoll ist, sind die Ergebnisse bereits laufender kontrollierter Studien noch ausstehend [65].

\section{Inhalative Immunsuppressiva}

Da der immunsuppressive Effekt dosisabhängig ist, stellt die inhalative Applikation von Immunsuppressiva einen naheliegenden Ansatz dar, um eine hohe lokale Konzentration der Medikamente im Transplantat, verbunden mit der Reduktion systemischer Nebenwirkungen, zu erreichen. In einigen unkontrollierten Studien konnte gezeigt werden, dass inhalatives Cyclosporin A bei therapierefraktärer akuter Abstoßung und progredientem BOS wirksam ist [66]. Darüber hinaus demonstrierte eine monozentrische, plazebokontrollierte, randomisierte Studie, dass inhalatives Cyclosporin als Prophylaxe zusätzlich zur StandardDreifachimmunsuppression zwar nicht die Rate akuter AbstoBungen reduziert, aber das BOS-freie Intervall und das Überleben signifikant verbesserte. Einschränkend ist allerdings anzumerken, dass über 50\% der Patienten sowohl im Verum- als auch im Plazebo-Arm die Studie insbesondere aufgrund von Infektionen und lokalen pulmonalen Reizungen vorzeitig beendeten [67]. Tierexperimentelle Daten zeigen, dass auch inhalatives Tacrolimus eine mögliche Option darstellt [68]. Durch Entwicklung einer liposomalen CyA-Formulierung und technische Verbesserungen in der Aerosoldeposition ist eine effektivere und nebenwirkungsärmere Applikation von inhalativem Cyclosporin möglich, sodass auch in diesem Bereich eine multizentrische, randomisierte, prospektive Studie initiiert wurde [69].

\section{Therapie der akuten zellulären Abstoßungsreaktion}

$\nabla$

Eine akute zelluläre Transplantatabstoßungsreaktion wird üblicherweise mit einer intravenösen Kortikosteroidstoßtherapie in einer Dosis von $500 \mathrm{mg}$ oder $1000 \mathrm{mg}$ Methylprednisolon pro Tag über drei bis fünf Tage behandelt. Hieran schließt sich normalerweise eine Erhöhung der oralen Prednisolontagesdosis auf 0,5 bis $1,0 \mathrm{mg} / \mathrm{kg}$ Körpergewicht mit schrittweiser Dosisreduktion über zwei bis drei Wochen an.

Für therapieresistente oder rezidivierende akute Abstoßungen kann bei Fehlen kontrollierter prospektiver Studien aufgrund der vorliegenden internationalen Erfahrungen eine Umstellung der Immunsuppression von Cyclosporin A auf Tacrolimus als erster Schritt empfohlen werden [70]. Für eine weitere Umstellung der Erhaltungstherapie von einem Calcineurin-Inhibitor auf Sirolimus oder von Azathioprin auf MMF gilt, dass es hierzu eine Reihe von unkontrollierten Untersuchungen gibt, ohne dass ein internationaler Konsens bestehen würde.

Nach der Umstellung der Immunsuppression von Cyclosporin A auf Tacrolimus ist die Therapie mit Substanzen, wie sie auch zur Induktionstherapie verwendet werden, in der Regel der zweite Schritt zur Therapie der rezidivierenden akuten Abstoßung. Eine weitere Therapieoption, die nach LTX bisher nicht formal untersucht wurde, für die es aber nach Nieren- und Herztransplantation günstige Berichte gibt, ist die nebenwirkungsarme intravenöse Gabe von hochdosierten Immunglobulinen [71].

Weitere Behandlungsmodalitäten umfassen, wie bereits erwähnt, inhalatives Cyclosporin A, Lymphsystembestrahlung (Total lymphoid irradiation) und die extrakorporale Photopherese (ExP), ohne dass hierzu ein größerer Erfahrungsschatz aus kontrollierten Studien vorliegen würde.

\section{Therapie der Bronchiolitis obliterans und des Bronchiolitis-obliterans-Syndroms}

Die Therapie der BO bzw. seiner klinisch-funktionalen Manifestation, dem BOS, bleibt die größte Herausforderung in der Langzeitnachsorge nach LTX. Zum jetzigen Zeitpunkt gibt es keine therapeutische Maßnahme, für die in kontrollierten Studien ein eindeutig positiver Effekt gezeigt werden konnte [72]:

1. Wechsel der Immunsuppression innerhalb therapeutischer Klassen. Hierzu gilt, dass die vorliegende Datenlage dies nur für einen Wechsel von Cyclosporin A auf Tacrolimus mit ausreichender Evidenz nahelegt.

2. Verstärkung der Immunsuppression durch Therapieansätze ähnlich der Induktionstherapie (s. o.).

3. Einsatz von inhalativem Cyclosporin A. Für dieses potenziell sehr erfolgversprechende Therapiekonzept laufen Studien, die auch einen prophylaktischen Einsatz untersuchen.

4. Lymphsystembestrahlung (Total lymphoid irradiation) und extrakorporale Photopherese (ExP) (s.o.).

5. Azithromycin (s.o.).

Das Konzept einer Intensivierung der Immunsuppression zur Therapie des BOS muss vor dem Hintergrund der mangelnden Effektivität und der gehäuft auftretenden, lebensbedrohlichen Infektionen kritisch hinterfragt werden. Da neuere Ansätze zur Pathogenese der BO zusätzlich zu immunologischen Faktoren insbesondere auch infektiöse Triggermechanismen diskutieren, setzen aktuelle Therapiestrategien an diesem Punkt an. Hier ist die Vermeidung bzw. Therapie von viralen Nicht-CMV-Infektio- 
nen der Lunge und insbesondere der Einsatz von antiinflammatorischen Medikamenten wie Azithromyzin zu erwähnen.

\section{Infektionsprophylaxe nach Lungentransplantation}

Infektiöse Komplikationen bleiben einer der wichtigsten Gründe für Morbidität und Mortalität nach LTX. Die Lunge selbst stellt dabei nach LTX das am häufigsten von Infekten betroffene Organ dar [1]. Gründe für diese Suszeptibilität sind der fortwährende Kontakt der Lungenoberfläche mit der Außenwelt, die Denervierung des Transplantats mit dem dadurch verminderten Hustenreflex und der gestörten Mukoziliärenclearance, Anastomosenkomplikationen, Übertragung von Keimen aus der Spenderlunge sowie aus der nativen Lunge bei Einzellungentransplantation auf das Transplantat. Mögliche Impfungen und angewendete präventive und prophylaktische Therapiestrategien für virale, mykotische und bakterielle Infekte werden im Folgenden erläutert.

\section{Impfung}

Im Vorfeld einer LTX und im Verlauf bei stabiler Transplantatfunktion werden die Durchführung von jährlichen Grippeschutzimpfungen und die Pneumokokken-Impfung empfohlen. Lebendimpfstoffe hingegen sind kontraindiziert. Insgesamt ist aber festzustellen, dass die Anwendung von Impfungen von den einzelnen Zentren sehr unterschiedlich gehandhabt wird. Darüber hinaus gibt es Hinweise, dass sowohl das Ausmaß als auch die Dauer der immunologischen Schutzwirkung einer Impfung nach Transplantation begrenzt sind [73-75].

\section{PCP-Prophylaxe}

Die Prävention einer Pneumocystis jirovecii-Pneumonie mit Cotrimoxazol (Sulfamethoxazol + Trimethoprim) ist hocheffektiv und bietet zusätzlich auch einen Schutz vor Toxoplasma gondiiInfektion sowie eine antimikrobielle Wirkung auf verschiedene Nocardien- und Listerien-Spezies. Sie ist daher Standard nach LTX und wird in den meisten Zentren dauerhaft durchgeführt $[73,76]$.

\section{CMV-Prophylaxe}

Cytomegalovirus (CMV) stellt das wichtigste virale Pathogen nach LTX dar. Eine CMV-Infektion ist eine wichtige Ursache für erhöhte Morbidität und Mortalität, insbesondere in der Frühphase nach LTX. Darüber hinaus ist eine durchgemachte CMV-Erkrankung aber auch mit einem erhöhten Risiko für BOS und damit einem reduzierten Langzeitüberleben assoziiert [1]. Grundsätzlich existieren zwei Therapiestrategien zur Verhinderung einer klinisch apparenten CMV-Infektion. Entweder die routinemäßige antivirale medikamentöse Prophylaxe oder aber die präemptive Therapie von Patienten, bei denen durch ein konsequentes Monitoring eine CMV-Virämie vor Ausbruch einer Organmanifestation detektiert wurde.

Unter Berücksichtigung auch der Erfahrungen mit anderen soliden Organtransplantationen lässt sich sagen, dass eine Prophylaxe mit Ganciclovir $\left(\right.$ Cymeven $^{\circledR}$ ), Valganciclovir $\left(\right.$ Valcyte $^{\circledR}$ ) oder Aciclovir die CMV-bedingte Mortalität senkt und auch Herpes zoster- und Herpes simplex-assoziierte Erkrankungen nach LTX reduziert. Diese Effekte gelten sowohl für CMV-seropositive als auch CMV-seronegative Empfänger von CMV-seropositiven Organen. Hierbei sind Ganciclovir und Valganciclovir untereinander gleichwertig und beide effektiver als Aciclovir einzuschätzen. Unklar bleiben jedoch die notwendige Dauer und die Dosierung der Prophylaxe, um einen möglichst optimalen Effekt bei klinisch relevanter Nephrotoxizität und myelosuppressiver Potenz dieser antiviralen Substanzen als Dauermedikation zu erzielen. Zusätzlich ist bei längerem Einsatz der Präparate mit einer relevanten Zunahme von Resistenzen zu rechnen [77,78].

\section{Antimykotische Prophylaxe}

Eine Pilzinfektion tritt in ca. 15\%-35\% der Fälle nach LTX auf. Häufigste Erreger sind hierbei Candida- und Aspergillus-Spezies, wobei insbesondere bei Candida eine Differenzierung zwischen echter Infektion und lediglich Kolonisierung der Atemwege häufig nicht sicher möglich ist. Für Aspergillus-Spezies gilt jedoch, dass auch eine Kolonisierung bereits einen Risikofaktor für eine spätere invasive Aspergillose, eine Anastomoseninfektion und besonders auch für die Entwicklung eines BOS darstellt. Daher wird insbesondere bei Patienten mit Risikomerkmalen (SingleLTX, chronische Niereninsuffizienz, CMV-Infektion, BOS) und in der frühen postoperativen Phase eine prophylaktische oder präemptive Therapie großzügig indiziert [79]. Die Wahl der eingesetzten Antimykotika (z.B. Itraconazol, Voriconazol, inhalatives Amphotericin B) und die Dosis sowie Dauer der Behandlung variieren dabei stark in Abhängigkeit vom jeweiligen Transplantationszentrum [80].

\section{Antibakterielle Prophylaxe}

Eine prophylaktische Breitspektrum-Antibiotikagabe insbesondere mit Abdeckung auch gramnegativer Erreger ist Teil des perioperativen Standards nach LTX. Die häufigsten Infektionen im Verlauf sind auf Pseudomonas aeruginosa zurückzuführen [81]. Darüber hinaus gilt analog zu Aspergillen-Spezies, dass eine nicht vorbestehende Kolonisierung mit P. aeruginosa als wichtiger Risikofaktor für eine BOS bei Patienten mit zystischer Fibrose identifiziert wurde [82]. Aus dieser Beobachtung ergibt sich die Rationale insbesondere zur inhalativen Behandlung (z.B. Colistin, Trobramycin) auch von asymptomatischen Patienten mit $P$. aeruginosa-Nachweis.

\section{Bisher erschienene Beiträge dieser Serie $\nabla$}

T. Schreder et al. Lungentransplantation bei Lungenemphysem Wer? Wann? Wie? Pneumologie 2010; 64: 632 -639

\section{Interessenkonflikt}

$\nabla$

Die Autoren geben an, dass kein Interessenkonflikt besteht.

\section{Literatur}

1 Christie JD, Edwards LB, Aurora P et al. The Registry of the International Society for Heart and Lung Transplantation: Twenty-sixth Official Adult Lung and Heart-Lung Transplantation Report-2009. J Heart Lung Transplant 2009; 28: 1031 - 1049

2 Palmer SM, Miralles AP, Lawrence CM et al. Rabbit antithymocyte globulin decreases acute rejection after lung transplantation: results of a randomized, prospective study. Chest 1999; 116: 127-133

3 Wiebe K, Harringer W, Wahlers T et al. ATG induction therapy and the incidence of bronchiolitis obliterans after lung transplantation: does it make a difference? Transplant Proc 1998; 30: 1517-1518

4 Wain JC, Wright CD, Ryan DP. Induction immunosuppression for lung transplantation with OKT3. Ann Thorac Surg 1999; 67: 187-193

5 Brock MV, Borja MC, Ferber L et al. Induction therapy in lung transplantation: a prospective, controlled clinical trial comparing OKT3, antithymocyte globulin, and daclizumab. J Heart Lung Transplant 2001; 20: $1282-1290$ 
6 Abramowicz D, Schandene L, Goldman M et al. Release of tumor necrosis factor, interleukin-2, and gamma-interferon in serum after injection of OKT3 monoclonal antibody in kidney transplant recipients. Transplantation 1989; 47: 606-608

7 Ding IB, Baumgartner RA, Schwaiblmair M, Behr J. Administration of anti-interleukin-2Ralpha monoclonal antibody in bronchiolitis obliterans syndrome after lung transplantation. Transplantation 2003; 75 : $1767-1769$

8 Burton CM, Andersen CB, Jensen AS et al. The incidence of acute cellular rejection after lung transplantation: a comparative study of anti-thymocyte globulin and daclizumab. J Heart Lung Transplant 2006; 25: $638-647$

9 Garrity ER Jr., Villanueva J, Bhorade SM et al. Low rate of acute lung allograft rejection after the use of daclizumab, an interleukin 2 receptor antibody. Transplantation 2001; 71: 773-777

10 McCurry KR, Iacono A, Zeevi A et al. Early outcomes in human lung transplantation with Thymoglobulin or Campath-1H for recipient pretreatment followed by posttransplant tacrolimus near-monotherapy. J Thorac Cardiovasc Surg 2005; 130: 528 - 537

11 van Loenhout KC, Groves SC, Galazka $M$ et al. Early outcomes using alemtuzumab induction in lung transplantation. Interact Cardiovasc Thorac Surg 2010; 10: 190-194 [Epub 2009 Nov 25]

12 Hoffmeyer F, Hoeper MM, Spiekerkotter E et al. Azathioprine withdrawal in stable lung and heart/lung recipients receiving cyclosporine-based immunosuppression. Transplantation 2000; 70: 522 - 525

13 Korom S, Boehler A, Weder W. Immunosuppressive therapy in lung transplantation: state of the art. Eur J Cardiothorac Surg 2009; 35: 1045 - 1055 [Epub 2009 Apr 5]

14 Borel JF, Feurer C, Gubler HU, Stahelin H. Biological effects of cyclosporin A: a new antilymphocytic agent. Agents Actions 1976; 6: 468-475

15 Levy G, Thervet E, Lake J, Uchida K. Consensus on Neoral C(2): Expert Review in Transplantation (CONCERT) Group. Patient management by Neoral C(2) monitoring: an international consensus statement. Transplantation 2002; 73 (9 Suppl): $12-18$

16 Nemati E, Einollahi B, Taheri S et al. Cyclosporine trough (c0) and 2hour postdose (c2) levels: which one is a predictor of graft loss? Transplant Proc 2007; 39: 1223-1224

17 Glanville AR, Morton JM, Aboyoun CL et al. Cyclosporine C2 monitoring improves renal dysfunction after lung transplantation. J Heart Lung Transplant 2004; 23: 1170-1174

18 Glanville AR, Aboyoun CL, Morton JM et al. Cyclosporine C2 target levels and acute cellular rejection after lung transplantation. J Heart Lung Transplant 2006; 25: 928-934

19 Taylor JL, Palmer SM. Critical care perspective on immunotherapy in lung transplantation. J Intensive Care Med 2006; 21: 327 - 344

20 Ragette R, Kamler M, Weinreich G et al. Tacrolimus pharmacokinetics in lung transplantation: new strategies for monitoring. J Heart Lung Transplant 2005; 24: 1315-1319

21 Knoop C, Thiry P, Saint-Marcoux F et al. Tacrolimus pharmacokinetics and dose monitoring after lung transplantation for cystic fibrosis and other conditions. Am J Transplant 2005; 5: 1477 - 1482

22 Reichenspurner $H$. Overview of tacrolimus-based immunosuppression after heart or lung transplantation. J Heart Lung Transplant 2005; 24: $119-130$

23 Keenan RJ, Konishi H, Kawai A et al. Clinical trial of tacrolimus versus cyclosporine in lung transplantation. Ann Thorac Surg 1995; 60: $580-584$

24 Treede H, Klepetko W, Reichenspurner H et al.; Munich and Vienna Lung Transplant Group. Tacrolimus versus cyclosporine after lung transplantation: a prospective, open, randomized two-center trial comparing two different immunosuppressive protocols. J Heart Lung Transplant 2001; 20: $511-517$

25 Zuckermann A, Reichenspurner H, Birsan T et al. Cyclosporine A versus tacrolimus in combination with mycophenolate mofetil and steroids as primary immunosuppression after lung transplantation: one-year results of a 2-center prospective randomized trial. J Thorac Cardiovasc Surg 2003; 125: $891-900$

26 Reichenspurner H, Glanville A, Christina A et al. and European and Australian Investigators in Lung Transplantation. Complete 3 Year Analysis of a Prospective Randomized International Multi-Center Investigator Driven Study Comparing Tacrolimus and Cyclosporin A, Both in Combination with MMF and Steroids after Lung Transplantation in 249 Patients. J Heart Lung Transplant 2008; 27: S205-S206
27 Hachem RR, Yusen RD, Chakinala MM et al. A randomized controlled trial of tacrolimus versus cyclosporine after lung transplantation. J Heart Lung Transplant 2007; 26: 1012 - 1018

28 Starnes VA. Heart-lung transplantation: an overview. Cardiol Clin 1990; 8: 159- 168

29 Azzola A, Havryk A, Chhajed P et al. Everolimus and mycophenolate mofetil are potent inhibitors of fibroblast proliferation after lung transplantation. Transplantation 2004; 77: 275-280

30 Kobashigawa J, Miller L, Renlund D et al. A randomized active-controlled trial of mycophenolate mofetil in heart transplant recipients Mycophenolate Mofetil Investigators. Transplantation 1998; 66: 507 515

31 Mathew TH. A blinded, long-term, randomized multicenter study of mycophenolate mofetil in cadaveric renal transplantation: results at three years. Tricontinental Mycophenolate Mofetil Renal Transplantation Study Group. Transplantation 1998; 65: 1450-1454

32 Palmer SM, Baz MA, Sanders $L$ et al. Results of a randomized, prospective, multicenter trial of mycophenolate mofetil versus azathioprine in the prevention of acute lung allograft rejection. Transplantation 2001; 71: $1772-1776$

33 McNeil K, Glanville AR, Wahlers T et al. Comparison of mycophenolate mofetil and azathioprine for prevention of bronchiolitis obliterans syndrome in de novo lung transplant recipients. Transplantation 2006; 81: $998-1003$

34 Wali RK, Mohanlal V, Ramos E et al. Early withdrawal of calcineurin inhibitors and rescue immunosuppression with sirolimus-based therapy in renal transplant recipients with moderate to severe renal dysfunction. Am J Transplant 2007; 7: 1572-1583

35 Fairbanks KD, Eustace JA, Fine D et al. Renal function improves in liver transplant recipients when switched from a calcineurin inhibitor to sirolimus. Liver Transpl 2003; 9: 1079-1085

36 Snell GI, Levvey BJ, Chin Wet al. Sirolimus allows renal recovery in lung and heart transplant recipients with chronic renal impairment. J Heart Lung Transplant 2002; 21: 540-546

37 Groetzner J, Wittwer T, Kaczmarek I et al. Conversion to sirolimus and mycophenolate can attenuate the progression of bronchiolitis obliterans syndrome and improves renal function after lung transplantation. Transplantation 2006; 81: 355-360

38 Venuta F, De Giacomo T, Rendina EA et al. Recovery of chronic renal impairment with sirolimus after lung transplantation. Ann Thorac Surg 2004; 78: $1940-1943$

39 Snell GI, Levvey BJ, Chin W et al. Rescue therapy: a role for sirolimus in lung and heart transplant recipients. Transplant Proc 2001; 33: 1084 1085

40 Ussetti P, Laporta R, de Pablo A et al. Rapamycin in lung transplantation preliminary results. Transplant Proc 2003; 35: 1974-1977

41 King-Biggs MB, Dunitz JM, Park SJ et al. Airway anastomotic dehiscence associated with use of sirolimus immediately after lung transplantation. Transplantation 2003; 75: 1437-1443

42 Groetzner J, Kur F, Spelsberg F et al.; Munich Lung Transplant Group. Airway anastomosis complications in de novo lung transplantation with sirolimus-based immunosuppression. J Heart Lung Transplant 2004; 23: $632-638$

43 Chhajed PN, Dickenmann M, Bubendorf $L$ et al. Patterns of pulmonary complications associated with sirolimus. Respiration 2006; 73: $367-$ 374

44 Bhorade SM, Ahya V, Kotloff R et al. Long Term Follow-Up in the AIRSAC Trial, a Multicenter Randomized Clinical Trial in Lung Transplant Recipients. J Heart and Lung Transplant 2009; 28: S119-S120

45 Neumayer $\mathrm{HH}$. Introducing everolimus (Certican) in organ transplantation: an overview of preclinical and early clinical developments. Transplantation 2005; 79: S72 - S75

46 Snell GI, Valentine VG, Vitulo P et al.; RAD B159 Study Group. Everolimus versus azathioprine in maintenance lung transplant recipients: an international, randomized, double-blind clinical trial. Am J Transplant 2006; 6: 169-177

47 Strueber M, Stefan F, Simon AR et al. Everolimus Versus Mycophenolatemofetil in De Novo Immunosuppression after Lung Transplantation - Interims Analysis of a Prospective, Randomized, Clinical Trial. J Heart and Lung Transplant 2008; 27: 205

48 Gullestad L, Iversen M, Mortensen SA et al. Everolimus with reduced calcineurin inhibitor in thoracic transplant recipients with renal dysfunction: a multicenter, randomized trial. Transplantation 2010; 89: $864-872$ 
49 Otton J, Hayward CS, Keogh AM et al. Everolimus-associated pneumonitis in 3 heart transplant recipients. J Heart Lung Transplant 2009; 28: 104-106 [Epub 2008 Dec 12]

50 Pratap B, Abraham G, Srinivas CN, Bhaskar S. Post-renal transplant hemolytic uremic syndrome following combination therapy with tacrolimus and everolimus. Saudi J Kidney Dis Transpl 2007; 18: 609612

51 Morton JM, Williamson S, Kear LM et al. Therapeutic drug monitoring of prednisolone after lung transplantation. J Heart Lung Transplant 2006; 25: $557-563$

52 Borro JM, Solé A, De la Torre $M$ et al. Steroid withdrawal in lung transplant recipients. Transplant Proc 2005; 37: 3991 - 3993

53 Shitrit D, Bendayan D, Sulkes J et al. Successful steroid withdrawal in lung transplant recipients: result of a pilot study. Respir Med 2005; 99: $596-601$

54 Keogh AM, Arnold RH, Macdonald PS et al. A randomized trial of tacrolimus (FK506) versus total lymphoid irradiation for the control of repetitive rejection after cardiac transplantation. J Heart Lung Transplant 2001; 20: 1331 - 1334

55 Diamond DA, Michalski JM, Lynch JP, Trulock EP. Efficacy of total lymphoid irradiation for chronic allograft rejection following bilateral lung transplantation. Int J Radiat Oncol Biol Phys 1998; 41: 795-800

56 Fisher AJ, Rutherford RM, Bozzino J et al. The safety and efficacy of total lymphoid irradiation in progressive bronchiolitis obliterans syndrome after lung transplantation. Am J Transplant 2005; 5: 537-543

57 Verleden GM, Lievens Y, Dupont LJ et al. Efficacy of total lymphoid irradiation in azithromycin nonresponsive chronic allograft rejection after lung transplantation. Transplant Proc 2009; 41: 1816-1820

58 Astor TL, Weill D. Extracorporeal photopheresis in lung transplantation. J Cutan Med Surg 2003; 7: 20-24

59 Morrell MR, Despotis GJ, Lublin DM et al. The efficacy of photopheresis for bronchiolitis obliterans syndrome after lung transplantation. J Heart Lung Transplant 2010; 29: 424-431 [Epub 2009 Oct 22]

60 Benden C, Speich R, Hofbauer GF et al. Extracorporeal photopheresis after lung transplantation: a 10-year single-center experience. Transplantation 2008; 86: 1625-1627

61 Williams TJ, Verleden GM. Azithromycin: a plea for multicenter randomized studies in lung transplantation. Am J Respir Crit Care Med 2005: 172: 657-659

62 Verleden GM, Dupont LJ. Azithromycin therapy for patients with bronchiolitis obliterans syndrome after lung transplantation. Transplantation 2004; 77: $1465-1467$

63 Vanaudenaerde BM, Meyts I, Vos R et al. A dichotomy in bronchiolitis obliterans syndrome after lung transplantation revealed by azithromycin therapy. Eur Respir J 2008; 32: 832-843

64 Gottlieb J, Szangolies J, Koehnlein T et al. Long-term azithromycin for bronchiolitis obliterans syndrome after lung transplantation. Transplantation 2008; 85: 36- 41

65 Vos R, Vanaudenaerdea BM, Schoonisa A et al. Azithromycin for Bronchiolitis Obliterans Syndrome after Lung Transplantation. J Heart and Lung Transplant 2010; 29: S94
66 Iacono AT, Corcoran TE, Griffith BP et al. Aerosol cyclosporin therapy in lung transplant recipients with bronchiolitis obliterans. Eur Respir J 2004; 23: $384-390$

67 Iacono AT, Johnson BA, Grgurich WF et al. A randomized trial of inhaled cyclosporine in lung-transplant recipients. N Engl J Med 2006; 354: $141-150$

68 Ide N, Nagayasu T, Matsumoto $K$ et al. Efficacy and safety of inhaled tacrolimus in rat lung transplantation. J Thorac Cardiovasc Surg 2007; 133: $548-553$

69 Behr J, Zimmermann G, Baumgartner R et al.; Munich Lung Transplant Group. Lung deposition of a liposomal cyclosporine A inhalation solution in patients after lung transplantation. J Aerosol Med Pulm Drug Deliv 2009; 22: 121 - 130

70 Sarahrudi K, Estenne $M$, Corris $P$ et al. International experience with conversion from cyclosporine to tacrolimus for acute and chronic lung allograft rejection. J Thorac Cardiovasc Surg 2004; 127: 1126 1132

71 Jordan SC, Quartel AW, Czer LS et al. Posttransplant therapy using highdose human immunoglobulin (intravenous gammaglobulin) to control acute humoral rejection in renal and cardiac allograft recipients and potential mechanism of action. Transplantation 1998; 66: 800-805

72 Bhorade SM, Stern E. Immunosuppression for lung transplantation. Proc Am Thorac Soc 2009; 6: 47-53

73 Fishman JA. Infection in solid-organ transplant recipients. N Engl J Med 2007; 357: $2601-2614$

74 Remund KF, Best $M$, Egan JJ. Infections relevant to lung transplantation. Proc Am Thorac Soc 2009; 6: 94-100

75 Avery RK, Michaels M. Update on immunizations in solid organ transplant recipients: what clinicians need to know. Am J Transplant 2008; 8: 9-14 [Epub 2007 Dec 18

76 Green H, Paul M, Vidal L, Leibovici L. Prophylaxis of Pneumocystis pneumonia in immunocompromised non-HIV-infected patients: systematic review and meta-analysis of randomized controlled trials. Mayo Clin Proc 2007; 82: 1052-1059

77 Hodson EM, Craig JC, Strippoli GF, Webster AC. Antiviral medications for preventing cytomegalovirus disease in solid organ transplant recipients. Cochrane Database Syst Rev 2008; 16: CD003774

78 Fishman JA, Emery V, Freeman R et al. Cytomegalovirus in transplantation - challenging the status quo. Clin Transplant 2007; 21: 149-158

79 Weigt SS, Elashoff RM, Huang $C$ et al. Aspergillus colonization of the lung allograft is a risk factor for bronchiolitis obliterans syndrome. Am J Transplant 2009; 9: 1903 - 1911 [Epub 2009 May 13]

80 Solé A, Salavert M. Fungal infections after lung transplantation. Curr Opin Pulm Med 2009; 15: $243-253$

81 Campos S, Caramori M, Teixeira $R$ et al. Bacterial and fungal pneumonias after lung transplantation. Transplant Proc 2008; 40: 822-824

82 Hafkin J, Blumberg E. Infections in lung transplantation: new insights. Curr Opin Organ Transplant 2009; 14: 483-487 